\title{
Low Recurrence Rate and Risk of Distant Metastases following Marginal Surgery of Intramuscular Lipoma and Atypical Lipomatous Tumors of the Extremities and Trunk Wall
}

\author{
Benjamin Presman Sune Frederik Jauffred Maj Raundrup Kornø \\ Michael Mørk Petersen \\ Musculoskeletal Tumor Section, Department of Orthopedic Surgery, Rigshospitalet, University of Copenhagen, \\ Copenhagen, Denmark
}

\section{Significance of the Study}

- A continued treatment strategy with marginal resection is considered justified for the treatment of intramuscular lipomas (IML) and atypical lipomatous tumors (ALT) based on the 10-year local recurrence-free survival rates of 95 and $81 \%$ for IML and ALT, respectively, absence of metastases, and only 2 dedifferentiation cases of atypical lipomatous tumors out of a total of 35 cases.

\section{Keywords}

Intramuscular lipoma - Atypical lipomatous tumors .

Local recurrence rate $\cdot$ Distant metastases

\begin{abstract}
Objective: The purpose of the present study was to determine the local recurrence rate, risk of dedifferentiation, and distant metastasis after surgical excision of intramuscular lipomas (IML) and atypical lipomatous tumors (ALT). Subjects and Methods: We retrospectively assessed all IML and ALT surgically removed from the extremities or trunk wall in our clinic between 1997 and 2006. Data from 141 patients with IML and 35 patients with ALT were extracted from the National Pathology Registry and patient files. Results: IML and ALT recurred in 10 and 6 tumors, respectively. No metastases were observed in either group. The 5- and 10-year local recurrence-free survival rates were $97.1 \%$ (94.3-99.9) and 94.8\% (Cl: 91.1-98.6) for IML and $84.6 \%(C l: 72.1-97.1)$ and
\end{abstract}

\begin{tabular}{ll}
\hline KARGER & $\begin{array}{l}\text { C) } 2019 \text { The Author(s) } \\
\text { Published by S. Karger AG, Basel }\end{array}$ \\
karger@karger.com & This is an Open Access article licensed under the Creative Commons \\
www.karger.com/mpp & $\begin{array}{l}\text { Attribution-NonCommercial-4.0 International License (CC BY-NC) } \\
\text { (http://www.karger.com/Services/OpenAccessLicense), applicable to } \\
\text { the online version of the article only. Usage and distribution for com- } \\
\text { mercial purposes requires written permission. }\end{array}$
\end{tabular}

81.1\% (Cl: 67.6-94.8) for ALT, respectively. ALT were found to dedifferentiate in 2/35 cases. Conclusion: Both IML and ALT showed a low recurrence rate when removed surgically from the extremities or trunk wall with intended marginal resection. No distant metastases were observed in any of the groups. It, therefore, seems safe to treat these tumors with marginal resection.

(c) 2019 The Author(s)

Published by S. Karger AG, Basel

\section{Introduction}

Lipoma is a benign tumor composed of mature adipocytes. The tumor often presents as a painless soft tissue mass occurring with a peak incidence at age 40-60 years [1]. Although most lipomas originate from subcutaneous fat, some are located under the fascia and are called deepseated lipomas [2]. If the lipomas occur within the skeletal muscle, they are named intramuscular lipomas (IML). 

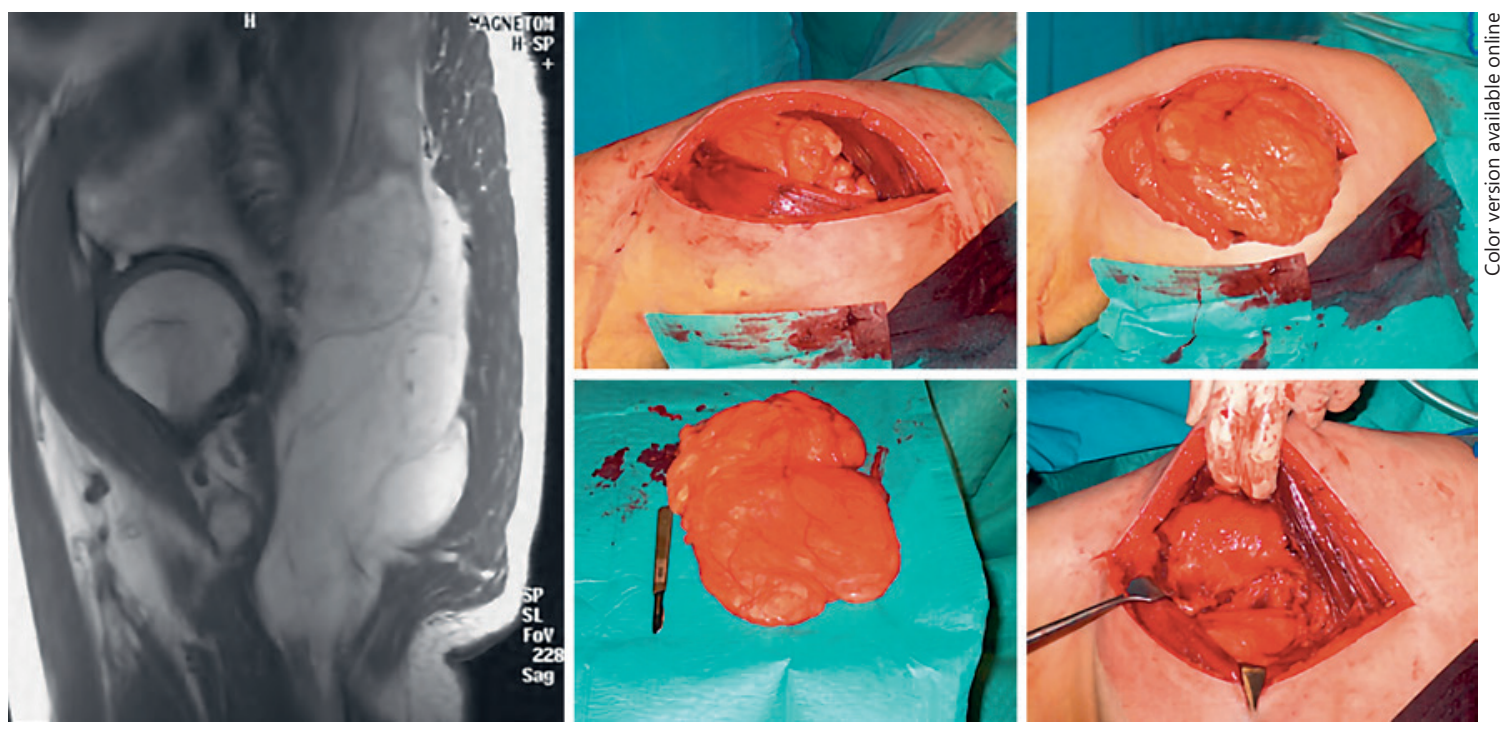

Fig. 1. An atypical lipomatous tumor of the gluteal region removed surgically with a margin. To the left, a T1weighted MRI (sagittal section) shows the tumor located below the gluteal muscles, and, to the right, various steps of marginal surgical tumor removal are shown.

IML are most often located in the large muscles of the extremities and trunk, but the exact topographical distribution varies among studies [2].

An atypical lipomatous tumor (ALT) of the extremities and trunk wall is a locally aggressive tumor. ALT was previously named well-differentiated liposarcomas, which constitute the largest subgroup of liposarcomas and account for $40-45 \%$. The lesion occurs most often in the sixth decade with an equal gender distribution. ALT and well-differentiated liposarcomas are synonyms with identical morphology and karyotype [1]. The term "welldifferentiated liposarcoma" is advocated by the WHO if the tumor is located in the retroperitoneum and mediastinum [1]. A complete resection of well-differentiated liposarcomas is often impossible due to their location, and even in the absence of dedifferentiation and metastasis, the tumor may ultimately lead to the patient's death [3]. Thus, the difference in behavior between ALT and welldifferentiated liposarcomas is solely related to the anatomical location and the possibility of performing a surgical resection [1].

Both IML, ALT, and well-differentiated liposarcomas are known to recur in some instances after surgical excision [3]. The purpose of this study was to determine the local recurrence rate, risk of dedifferentiation, and the occurrence of distant metastases after surgical excision of IML and ALT in the extremities and trunk wall.

\section{Subjects and Methods}

This was a retrospective study in which we retrieved data from our local pathology database and patient records on all patients with ALT or IML located in the extremities or trunk wall and surgically removed at our clinic between 1997 and 2006. The diagnosis of ALT and IML was done by experienced pathologists before the routine use of the MDM2 gene in the diagnostic process. MDM2 is a gene located on chromosome 12 and considered a highly sensitive marker for ALT [4]. However, at the time of primary diagnosis in this study, MDM2 was not used as a diagnostic tool to differentiate between the entities.

Only patients with primary tumors operated on for the first time were included in the study. In general, the surgical objective was to remove all tumors with marginal resection (Fig. 1). However, in case of tumor infiltration between muscle fibers, some healthy muscle tissue was removed with the tumor (according to the histology reports), and in some cases the tumors were removed with intralesional resection. Since the evaluation by the surgeon on whether the resection was done macroscopically marginal or intralesional could not always clearly be determined from the patient record, it is not recorded in the present paper. Patients with IML were discharged after surgery and had no follow-up in our facility apart from the early postoperative control involving suture removal and wound care. Patients with ALT were followed up and screened for recurrences with clinical controls and repeated magnetic resonance imaging (MRI) 5 years following surgery. Regardless of the diagnosis, all patients were controlled for recurrences via data obtained from the National Pathology Registry (NPR) in June 2017 [5]. NPR holds data of all pathological tissues removed at any hospital or private clinic in Denmark. The time of registered local recurrences was set from the time of primary operation to the 
Table 1. Baseline data and local recurrence data of patients with atypical lipomatous tumors (ALT) and intramuscular lipomas (IML)

\begin{tabular}{|c|c|c|c|}
\hline & ALT & IML & $\begin{array}{l}p \text { value (Mann- } \\
\text { Whitney U test) }\end{array}$ \\
\hline Patients, $n$ & 35 & 141 & \\
\hline Gender, females/males & $17 / 18$ & $69 / 72$ & \\
\hline Mean age (range), years & $59(34-85)$ & $54(3-105)$ & 0.028 \\
\hline Mean tumor size (range), $\mathrm{cm}$ & $15.1(3.0-45.0)$ & $7.5(1.7-25.0)$ & $<0.0005$ \\
\hline \multicolumn{4}{|l|}{ Tumor location I } \\
\hline Extremity: lower/upper & $25 / 7$ & $57 / 60$ & \\
\hline \multicolumn{4}{|l|}{ Tumor location II } \\
\hline Other: trunk wall/unknown & $3 / 0$ & $21 / 3$ & \\
\hline Local recurrence, $n$ & $6(17 \%)$ & $10(7 \%)$ & \\
\hline \multicolumn{4}{|l|}{ Mean time (range), months } \\
\hline To local recurrence & $36(7-56)$ & $88(8-168)$ & 0.056 \\
\hline From surgery to death & $88(0-213)$ & $108(14-220)$ & \\
\hline Follow-up & $144(0-240)$ & $160(14-248)$ & \\
\hline
\end{tabular}

date of the surgical re-resection. Survival data were extracted in June 2017 from the Danish Centralized Civil Register, which holds records of all deaths occurring in Denmark [6].

We included 35 patients ( 17 females and 18 males, mean age 59 years, range 34-85 years) with ALT and 141 patients (69 females and 72 males, mean age 54 years, range 3-105 years) with IML. Tumors were primarily located on the lower extremities $(n=25)$ in the ALT group. The remainder were distributed on the upper extremities $(n=7)$ and thoracic or abdominal wall $(n=3)$. The IML were more evenly distributed on the body with the majority of tumors located on the upper extremities $(n=60)$, lower extremities $(n=57)$, and the thoracic or abdominal wall $(n=21)$. Three IMLs were removed from unspecified areas (Table 1). Tumor size was expressed as the largest diameter. The ALT measured on average $15.1(3.0-45.0) \mathrm{cm}$, whereas the IML were generally smaller, measuring $7.5(1.7-25.0) \mathrm{cm}$ (Table 1).

Of the 29 disease-free ALT patients, 23 returned regularly for clinical and MRI follow-up in our outpatient facility for an average of 51 (6-126) months following surgery. One patient died in the perioperative period, and the remaining patients were lost to clinical and MRI follow-up for unknown reasons. All 35 patients were checked for local recurrences in the NPR. Average follow-up, i.e., the time from primary surgery to the occurrence of death or follow-up in NPR, was 144 (0-240) months.

Average time from surgery to death was 108 (14-220) months, with an average patient age of 75 (52-98) years in the IML group. There were no indications that these deaths were related to the IML disease. We did not follow these patients in our clinic on a regular basis. Local recurrences were found by reviewing the NPR. Mean follow-up of IML patients was 160 (14-248) months, i.e., the time from primary surgery to the time of the review in the NPR or occurrence of death. We found no unknown local recurrences in the NPR, thus indicating that all local recurrences were referred back to us in case a patient noticed disease recurrence. As in the ALT group, no distant metastases were recorded in the IML group.

Recurrence Rate and Risk of Metastases of

Benign Lipomatous Tumors
We used Kaplan-Meier survival analysis for the evaluation of 5- and 10-year local recurrence-free survival (LRFS) and patient overall survival (OS). Five- and 10-year survival data are presented with 95\% confidence intervals (CI) calculated using Greenwood's formula for the calculation of standard errors, and ALT and IML patients were compared using the log-rank test. Comparisons of age, tumor size, and time to local recurrence between groups were performed using a nonparametric test for unpaired data (MannWhitney $U$ test). $p$ values $<0.05$ were considered statistically significant. All descriptive data are given as means (total range). Data analyses were performed using IBM SPSS Statistics 22 and Microsoft Excel 2013. The study was approved by the Danish Data Protection Agency (file numbers: 2011-41-6596/2013-41-2371).

\section{Results}

In the ALT group, 6 of 35 patients had a local recurrence during the follow-up period, and recurrences were observed on average $36(7-73)$ months after the primary operation (Table 1). This corresponded to a 5-year LRFS of $84.6 \%$ (CI: 72.0-97.1) and a 10-year LRFS of $81.1 \%$ (CI: 67.6-94.8) (Fig. 2). Histology showed that the recurrence was unchanged in 4 patients, and dedifferentiation of liposarcomas was noted in 2 patients; the probability of avoiding dedifferentiation to a highly malignant sarcoma was 100 and $92.3 \%$ (CI: 83.1-100) after 5 and 10 years, respectively.

No distant metastases were registered in the ALT group, but at the end of the study, 14 patients had died on average $88(0-213)$ months after primary surgery, with an 
Fig. 2. Probability of local recurrence-free survival after surgical tumor excision of atypical lipomatous tumors (a) and intramuscular lipomas (b).

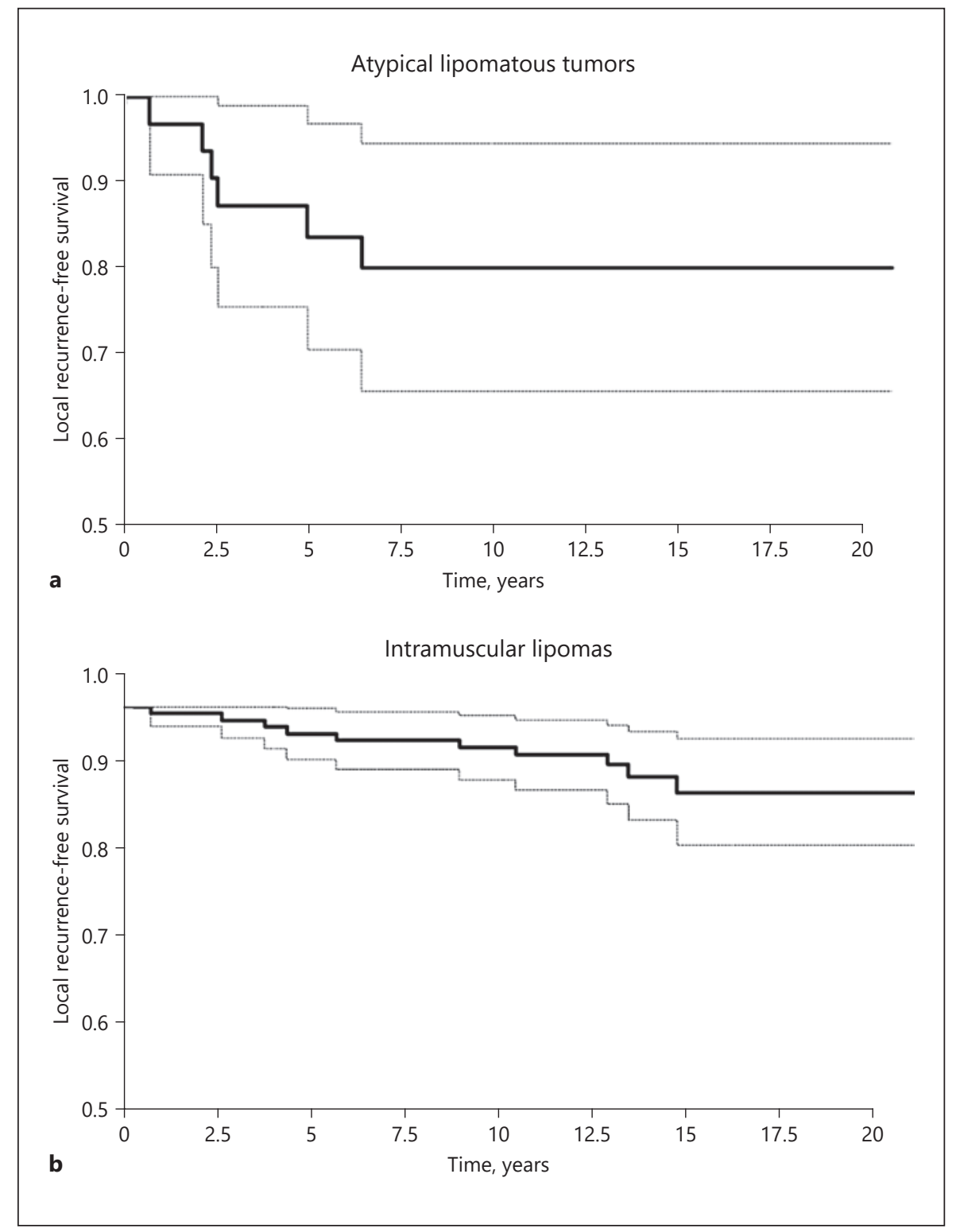

average age of 76 (54-95) years. This corresponds to a 5 -year OS of $85.7 \%$ (CI: 74.1-97.3) and a 10-year OS of $68.6 \%$ (CI: 53.2-83.9) following surgery (Fig. 3). None of the deaths in the ALT group were suspected to be related to the primary disease (ALT) or a local recurrence.

In the IML group, 10 patients were found to have local recurrences out of a total of 141 patients (Table 1). The 5- and 10-year LRFS for IML was 97.1\% (CI: 94.3-99.9) and $94.8 \%$ (CI: 91.1-98.6), respectively (Fig. 2). LRFS were observed on average 88 (8-168) months after the primary operation, and histology at recurrence was IML in 6 patients, while 4 tumors were diagnosed as ALT on the time of recurrence. Of the 141 IML patients, 25 died during the study period. This corresponds to a 5- and 10year OS of $95.0 \%$ (CI: 91.4-98.6) and 90.0\% (CI: 85.195.0) (Fig. 3).

Comparing LRFS and OS between patients treated for IML and ALT, we found that both the probability of LRFS $(p=0.02)$ and OS $(p=0.005)$ was higher in the IML group. The patients of the ALT group were older than those of the IML group $(p=0.02)$, and their tumors were substantially larger $(p<0.0005)$, with a tendency for the local recurrence to appear faster $(p=0.056)$ (Table 1$)$. 
Fig. 3. Probability of overall patient survival after surgical tumor excision of atypical lipomatous tumors (a) and intramuscular lipomas (b).

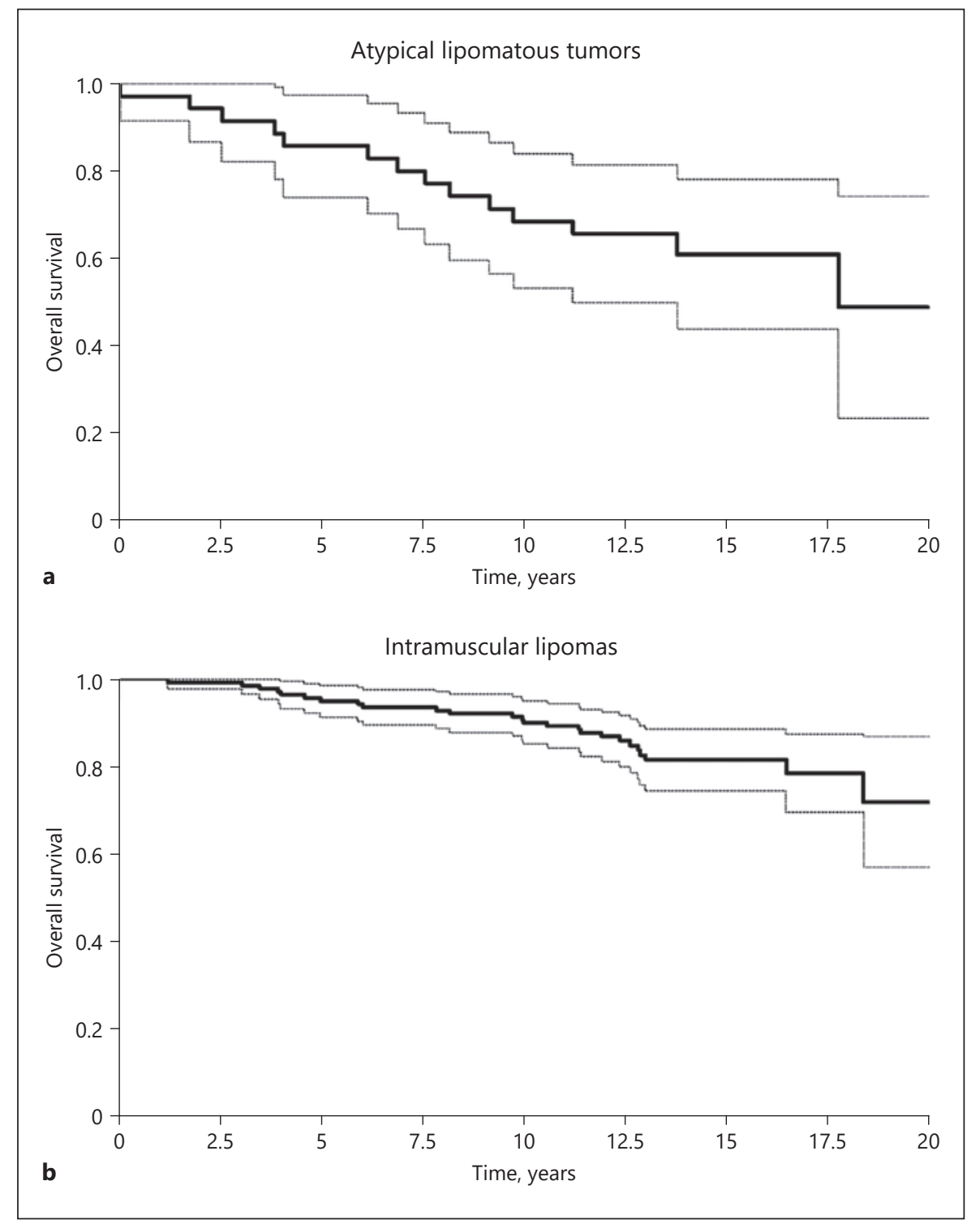

\section{Discussion}

ALT and IML are differential diagnoses. In this study, the ALT and IML diagnoses were made by experienced pathologists. Despite a histological overlap, ALT can be distinguished from IML by the presence of adipocytic proliferation with nuclear atypia in both adipocytes and stromal cells [1]. The presence of lipoblasts and thicker fibrous septae may further confirm the diagnosis [1]. Furthermore, the distinction between IML and ALT on MRI scans is at times challenging due to similar characteristics [7]. On MRI scans, IML and other simple lipomas tend to be smaller in size with thinner septae and less multilobu- lation compared to their more aggressive counterpart ALT [2]. Imaging features suggestive of ALT include tumor size $>10 \mathrm{~cm}$, thick irregular septae, nonadipose areas, and a fat content $<75 \%$ [7]. Other differential diagnoses include intramuscular hemangioma and primary muscular diseases. However, these differential diagnoses are easier to confirm or dismiss on MRI by exposing either the vascular component or the muscular dystrophic changes [2].

In recent years, the diagnosis of ALT versus IML has been based on molecular abnormality in ALT, namely a MDM2 gene amplification on chromosome 12 (12q1315), which helps to differentiate ALT from IML [4], but 
Fig. 4. T1-weighted MRI pictures (axial sections) at diagnosis in November 2002 of an intramuscular lipoma of the right forearm (a) and at local recurrence in October 2010 as an atypical lipomatous tumor (b). The micrographs show the tumor in 2002 (c): typical intramuscular lipoma infiltrating skeletal muscle (red cells; color refers to online version only), without atypia or any other signs of malignancy. The recurrence in 2010 (d) is still without any discernable atypia of lipocytes, but fibrous areas have appeared with a few spindle-shaped stromal cells (pink-gray areas; color refers to the online version only). Suspicion of atypical lipoma is confirmed by positive nuclear immunohistochemical staining for MDM2 in a few tumor cells (e).


at the time of the primary diagnosis in this study, MDM2 was not employed as a diagnostic tool to differentiate between both entities. This is a limitation of our study, but other studies have shown a $96 \%$ coherence between the initial histological diagnosis of ALT and the subsequent molecular confirmation. In a study by Zhang et al. [4], who performed a molecular cytogenetic evaluation of 405 tumors, only $4 \%$ of ALT tumors were reclassified to ordinary lipomas and only $1.7 \%$ from lipoma to ALT. It is plausible that the 4 IML cases showing recurrence and dedifferentiation to ALT in the present study were in fact primarily ALT, and that these patients were simply misclassified in the beginning. In Figure 4, MRI and histology of 1 of the 4 IML cases that recurred as an ALT are depicted. To further explore this issue, we did a recalculation of local recurrence rates for ALT and IML with the 4 IML patients that experienced a local recurrence as an ATL reclassified as ATL tumors. We found that for ALT, 5- and 10-year LRFS was decreased from 84.6 to $83.6 \%$ and from $81.1 \%$ to $70.6 \%$ respectively, while 5- and 10 - year LRFS for IML increased to $97.8 \%$ from originally 97.1 and $94.8 \%$, respectively. Thus, the 10 -year LRFS was mainly affected during this theoretical recalculation that showed the worst-case scenario of the influence of not using MDM2 in the initial diagnosis.

The recurrence rates for IML have shown great variance in the literature (3-62.5\%) [2]. When interpreting previous studies, it is important to keep in mind that it was previously mandatory to find lipoblasts in order to make the diagnosis of ALT [2]. Hence, the diagnosis would most likely have been IML if the histology showed no occurrence of lipoblasts. Thus, some older studies might have previously misdiagnosed ALT as IML due to missing lipoblasts. Both entities have different rates of local disease recurrence, and this might in part explain the recurrence rate widely reported in the literature. In our study, we did not have radiological or clinical follow-up of the IML group to assess the recurrence rate. However, NPR holds data of all pathological tissue removed at any hospital or private clinic in Denmark. Furthermore, a tu- 
mor recurrence would most probably be referred back to us for surgical re-resection. In the unlikely event that a patient with a recurrence was transferred to another hospital or private clinic in Denmark, the pathology record would appear in the NPR and would, therefore, also become known to us. This method diminishes the possibility of missing a recurrence. The number of patients refraining from surgical re-resection of IML recurrence is unknown. We can only speculate that most patients with a clinically relevant recurrence would be urged to seek medical attention. The true recurrence rate of IML might, therefore, be slightly larger than indicated in our results. Potential tumors that we may have missed on this account are most likely small and of less clinical importance, as the patients otherwise would probably have sought probably medical attention. Our study correlates well with previous studies showing a low risk of IML recurrence [2].

Studies concerning ALT recurrence rates are more often reported in the literature, and as with IML these studies have also shown great variance, ranging from 7.5$22.6 \%$ [8-12]. Only a few of these studies calculate a proper LRFS (89-95\%) after 5 years [11, 12], and only 1 study also calculated a 10-year LRFS of $82 \%$ [11], which is in good agreement with our ALT findings. The variance in the reported recurrence rates across the literature might in part be explained by the follow-up regimens used. Indeed, follow-up regimens vary widely among the centers in previously published materials [13]. Given that these are slow-growing tumors, we believe that a long-term follow-up is mandatory in order to properly estimate the local recurrence rate. In the present study, the mean follow-up was relatively long; for ALT 144 months (0-240) and for IML 160 months (14-248). The shortest follow-up in the groups was conducted on patients who died soon after surgery. Contrary to IML patients, all ALT patients were followed up several years after surgery with repeated MRI. At the end of the radiological follow-up period, all ALT patients were further controlled for recurrences in the NPR, in the same manner as for the IML, for the rest of the study period. These measures increase the likelihood of discovering any recurrence in this group. Although this probably identifies the vast majority of recurrences, we cannot completely rule out the possibility of missing some. Patients may choose to ignore symptoms of recurrence after the follow-up period. Although this may be the case for a proportion of patients in the IML group as discussed above, it seems much less likely for the ALT group. These latter patients were informed of having had removed a mild

Recurrence Rate and Risk of Metastases of Benign Lipomatous Tumors form of cancer (the term well-differentiated liposarcoma is still widely being used for these tumors in Denmark), so any signs of recurrence would most likely guide them to contact the hospital. In both groups, the possibility of patients leaving the country and experiencing a recurrence later remains. We do not anticipate this to be a significant problem, as very few patients in this age group are expected to emigrate.

The European Sarcoma Network Working Group recommends removing ALT tumors with marginal resection when located extracompartmentally [14]. However, in the literature, there is no consensus regarding the best surgical treatment for ALT. Thus, some clinics prioritize definitive therapy and, therefore, recommend a wide resection, while other clinics emphasize the low malignancy potential and recommend a marginal resection [13]. It is plausible that the variance in recurrence rates might also be explained by differences in the surgical techniques [15]. Kito et al. [15] published a study comparing wide resection with the traditional marginal excision for ALT of the extremities. They were able to show a $0 \%(0 / 11)$ recurrence rate for the widely resected tumors, whereas the marginally excised recurred in $23 \%(7 / 30)$ of the cases. In order to reduce our rate of recurrence comparably to their excellent results, we would have to adopt the wide excision strategy. This would imply resecting the tumors with a larger margin of healthy tissue and, in some cases, resecting whole muscle groups, which would render the procedure much more debilitating for the patient. However, this study confirms the results of all previous studies which have found no cases of distant metastasis or deaths directly related to ALT located in the extremities or trunk wall. As a consequence, it seems unnecessary to remove these tumors with any kind of margin that could result in debilitation.

Our study is in line with previous studies that have shown a very limited capability of ALT to dedifferentiate. The literature indicates that dedifferentiation occurs in $<2 \%$ of ALT lesions in the extremities [1]. In line with previous studies, none of the patients showed signs of distant metastases, and we found no ALT-related mortality. Consequently, the question of whether dedifferentiation is of any clinical importance to ALT remains to be answered.

\section{Conclusion}

We found high 10-year LRFS of 95 and $81 \%$ for IML and ALT, respectively. Our local rates of IML recurrence are comparable to earlier studies, and the ALT results cor- 
respond to the average results found in previously published articles. Both local rates of recurrence were found to be acceptably low given the benign nature of both disease entities. With no propensity to distant metastases and death in the present study and (to our knowledge) in previous studies, the applied surgical strategy of marginal excision seems to be most suitable for both IML and ALT.

\section{References}

1 Fletcher CD, Bridge JA, Hogendoom PC, Mertens F. WHO classification of tumours of soft tissue and bone. World Health Organization classification of tumours. 4th ed. Lyon: IARC Press; 2013.

2 McTighe S, Chernev I. Intramuscular lipoma: a review of the literature. Orthop Rev (Pavia). 2014 Dec;6(4):5618.

3 Bassett MD, Schuetze SM, Disteche C, Norwood TH, Swisshelm K, Chen X, et al. Deepseated, well differentiated lipomatous tumors of the chest wall and extremities: the role of cytogenetics in classification and prognostication. Cancer. 2005 Jan;103(2):409-16.

4 Zhang H, Erickson-Johnson M, Wang X, Oliveira JL, Nascimento AG, Sim FH, et al. Molecular testing for lipomatous tumors: critical analysis and test recommendations based on the analysis of 405 extremity-based tumors. Am J Surg Pathol. 2010 Sep;34(9): 1304-11.

5 Bjerregaard B, Larsen OB. The Danish Pathology Register. Scand J Public Health. 2011 Jul;39(7 Suppl):72-4.

6 Schmidt M, Pedersen L, Sørensen HT. The Danish Civil Registration System as a tool in epidemiology. Eur J Epidemiol. 2014 Aug; 29(8):541-9.
7 Brisson M, Kashima T, Delaney D, Tirabosco $\mathrm{R}$, Clarke A, Cro S, et al. MRI characteristics of lipoma and atypical lipomatous tumor/ well-differentiated liposarcoma: retrospective comparison with histology and MDM2 gene amplification. Skeletal Radiol. 2013 May; 42(5):635-47.

8 Errani C, Cocchi S, Ali N, Chehrassan M, Righi A, Gambarotti M, et al. Recurrence after marginal excision for atypical lipomatous tumors versus lipomas of the extremities. Orthopedics. 2016 Jul;39(4):e610-4.

9 Yamamoto N, Hayashi K, Tanzawa Y, Kimura $\mathrm{H}$, Takeuchi A, Igarashi $\mathrm{K}$, et al. Treatment strategies for well-differentiated liposarcomas and therapeutic outcomes. Anticancer Res. 2012 May;32(5):1821-5.

10 Fisher SB, Baxter KJ, Staley CA 3rd, Fisher KE, Monson DK, Murray DR, et al. The General Surgeon's quandary: atypical lipomatous tumor vs lipoma, who needs a surgical oncologist? J Am Coll Surg. 2013 Nov;217(5): 881-8.
11 Mussi CE, Daolio P, Cimino M, Giardina F, De Sanctis R, Morenghi E, et al. Atypical lipomatous tumors: should they be treated like other sarcoma or not? Surgical consideration from a bi-institutional experience. Ann Surg Oncol. 2014 Dec;21(13):4090-7.

12 Rauh J, Klein A, Baur-Melnyk A, Knösel T, Lindner L, Roeder F, et al. The role of surgical margins in atypical lipomatous tumours of the extremities. BMC Musculoskelet Disord. 2018 May;19(1):152.

13 Sommerville SM, Patton JT, Luscombe JC, Mangham DC, Grimer RJ. Clinical outcomes of deep atypical lipomas (well-differentiated lipoma-like liposarcomas) of the extremities. ANZ J Surg. 2005 Sep;75(9):803-6.

14 ESMO/European Sarcoma Network Working Group. Soft tissue and visceral sarcomas: ESMO Clinical Practice Guidelines for diagnosis, treatment and follow-up. Ann Oncol. 2014 Sep;25 Suppl 3:iii102-12.

15 Kito M, Yoshimura Y, Isobe K, Aoki K, Momose T, Suzuki S, et al. Clinical outcome of deep-seated atypical lipomatous tumor of the extremities with median-term follow-up study. Eur J Surg Oncol. 2015 Mar;41(3): 400-6. 